\title{
Central Sensitization of Mechanical Nociceptive Pathways Is Associated with a Long-Lasting Increase of Pinprick-Evoked Brain Potentials
}

\author{
Emanuel N. van den Broeke ${ }^{+*}$, Julien Lambert ${ }^{\dagger}$, Gan Huang and André Mouraux \\ Institute of Neuroscience, Université Catholique de Louvain, Brussels, Belgium
}

Intense or sustained nociceptor activation, occurring, for example, after skin injury, can induce "central sensitization," i.e., an increased responsiveness of nociceptive neurons in the central nervous system. A hallmark of central sensitization is increased mechanical pinprick sensitivity in the area surrounding the injured skin. The aim of the present study was to identify changes in brain activity related to this increased pinprick sensitivity. In 20 healthy volunteers, increased pinprick sensitivity was induced using high frequency electrical stimulation of the forearm skin (HFS). Mechanical pinprick stimulation (64 and $90 \mathrm{mN}$ ) was used to elicit event-related brain potentials (ERPs). The recordings were performed before, 20 min after and 45 min after applying HFS. The contralateral non-sensitized arm served as control. Pinprick stimulation of $64 \mathrm{mN}$, but not $90 \mathrm{mN}$,

OPEN ACCESS

Edited by:

Tetsuo Kida,

National Institute for Physiological

Sciences, Japan

Reviewed by:

Enrico Schulz,

University of Oxford, UK Andrea Truini,

Sapienza University of Rome, Italy

${ }^{*}$ Correspondence:

Emanuel N. van den Broeke emanuel.vandenbroeke@uclouvain.be

${ }^{\dagger}$ These authors have contributed equally to this work.

Received: 22 August 2016 Accepted: 05 October 2016 Published: 20 October 2016

Citation:

van den Broeke EN, Lambert J, Huang G and Mouraux A (2016) Central Sensitization of Mechanical Nociceptive Pathways is Associated with a Long-Lasting Increase of

Pinprick-Evoked Brain Potentials.

Front. Hum. Neurosci. 10:531.

doi: 10.3389/fnhum.2016.00531 applied in the area of increased pinprick sensitivity elicited a significant increase of a late-latency positive wave, between 300 and $1100 \mathrm{~ms}$ after stimulus onset and was maximal at midline posterior electrodes. Most importantly, this increase in EEG activity followed the time course of the increase in pinprick perception, both being present 20 and 45 min after applying HFS. Our results show that the central sensitization of mechanical nociceptive pathways, manifested behaviorally as increased pinprick sensitivity, is associated with a long-lasting increase in pinprick-evoked brain potentials provided that a $64 \mathrm{mN}$ stimulation intensity is used.

Keywords: central sensitization, hyperalgesia, mechanical, pinprick, evoked potentials, brain

\section{INTRODUCTION}

Injury to the skin leads to increased pain sensitivity of the injured skin ("primary hyperalgesia") but also of the surrounding uninjured skin ("secondary hyperalgesia"). A hallmark of secondary hyperalgesia is increased sensitivity to mechanical nociceptive stimuli such as pinprick stimuli (Raja et al., 1984; Ali et al., 1996; Magerl et al., 1998). The development of this increased pinprick sensitivity does not necessarily require tissue injury, as it can also be induced experimentally by activating nociceptors in a sustained and intense fashion, for example, using high frequency electrical stimulation of the skin (Henrich et al., 2015) or intradermal injection of capsaicin (LaMotte et al., 1991; Ziegler et al., 1999; Magerl et al., 2001). Importantly, the increase in pinprick sensitivity in the area of secondary hyperalgesia is thought to predominantly result from central sensitization (Baumann et al., 1991; LaMotte et al., 1991; Simone et al., 1991), defined by the International Association for the Study of Pain (IASP) as "increased responsiveness of nociceptive neurons in the central nervous system to their normal or subthreshold afferent input." 
In an attempt to explore the changes in brain activity related to this increased pinprick sensitivity, we recently recorded event-related brain potentials (ERPs) to mechanical pinprick stimulation of the skin before and after intradermal capsaicin injection (van den Broeke et al., 2015). We found that pinprick stimulation elicits both an early-latency negativepositive complex, maximal at the scalp vertex, and a later longlasting positive wave, maximal at more posterior brain regions. When the pinprick stimuli were applied to the area of secondary hyperalgesia, only the magnitude of this late positivity was significantly increased.

However, we also showed recently that, in addition to inducing increased pinprick sensitivity, HFS also induces an enhancement of the brain responses to thermal and innocuous tactile stimuli (van den Broeke and Mouraux, 2014a,b). These after-effects were observed $20 \mathrm{~min}$ after applying HFS, but were no longer present $45 \mathrm{~min}$ after HFS. This was in striking contrast with the robust increase in pinprick sensitivity that was observed both 20 and $45 \mathrm{~min}$ after HFS, and is known to last up to several hours (Pfau et al., 2011). Taken together, this indicates that the intense and sustained activation of nociceptors triggers distinct mechanisms: nociceptive-specific mechanisms generating a long-lasting increase in pinprick sensitivity and unspecific mechanisms generating a short-lasting enhancement of the brain responses to a variety of sensory stimuli.

Therefore, in order to determine whether the increase in magnitude of the late positive wave of pinprick-evoked potentials (PEPs) is somehow related to the phenomenon of mechanical hyperalgesia, it is crucial to demonstrate that this increase in brain response follows the same time course as the increase in pinprick sensitivity, i.e., that it is also present $45 \mathrm{~min}$ after applying HFS. This constituted the aim of the present study.

\section{MATERIALS AND METHODS}

\section{Participants}

Twenty healthy volunteers took part in the experiment ( 8 men and 12 women; aged $20-27$ years; $22.6 \pm 2.0$ years [mean \pm SD]). The experiments were conducted according to the Declaration of Helsinki. Approval for the experiment was obtained from the local Ethical Committee (Hospital and Departmental Ethics Committee, Saint-Luc - Université catholique de Louvain). All participants signed an informed consent form and received financial compensation for their participation.

\section{Experimental Design}

The design of the experiment is summarized in Figure 1. During the experiment, participants were comfortably seated in a chair with their arms resting on a table in front of them. Measurements were performed before (T0), $20 \mathrm{~min}$ after (T1) and $45 \mathrm{~min}$ after (T2) applying HFS to the left or right volar forearm. To avoid any confounding effect of handedness, the arm onto which HFS was applied (dominant vs. non-dominant) was counterbalanced across participants. Handedness was assessed using the Flinders Handedness Survey (Nicholls et al., 2013). For each measurement (T0, T1, T2), mechanical pinprick stimuli were applied to the skin adjacent to the area onto which HFS was delivered, and to the corresponding skin area of the contralateral arm which served as control. Two intensities of pinprick stimulation were used (64 and $90 \mathrm{mN}$ ). Each intensity was applied to a different skin site, distal or proximal from the site onto which HFS was delivered. This was balanced across participants, as well as the order of presentation of the two pinprick intensities, and the arm onto which stimuli were first applied (HFS vs. control arm).

\section{Induction of Increased Pinprick Sensitivity}

Transcutaneous high frequency electrical stimulation (HFS) was used to induce increased pinprick sensitivity in the surrounding unconditioned skin. The stimulation was applied to the volar forearm, $10 \mathrm{~cm}$ distal to the cubital fossa and consisted of five trains of $100 \mathrm{~Hz}$ (pulse width: $2 \mathrm{~ms}$ ) lasting $1 \mathrm{~s}$ each. The time interval between each train was $10 \mathrm{~s}$. The intensity of stimulation was individually adjusted to $20 \times$ the detection threshold to a single pulse $(0.28 \pm 0.09 \mathrm{~mA}$; mean $\pm \mathrm{sd})$. The electrical pulses were triggered by a programmable pulse generator (Master8; AMPI Israel), produced by a constant current electrical stimulator (Digitimer DS7A, Digitimer UK), and delivered to the skin using a specifically designed electrode designed and built at the Centre for Sensory-Motor Interaction (Aalborg University, Denmark). The cathode consists of 16 blunt stainless steel pins with a diameter of $0.2 \mathrm{~mm}$ protruding $1 \mathrm{~mm}$ from the base. The 16 pins are placed in a circle with a diameter of $10 \mathrm{~mm}$. The anode consists of a surrounding stainless steel ring having an inner diameter of $22 \mathrm{~mm}$ and an outer diameter of $40 \mathrm{~mm}$.

\section{Mechanical Pinprick Stimulation}

A custom-built device was used to deliver calibrated mechanical pinprick stimuli (Figure 2A). The stimulator consists of a cylindrical stainless steel flat tip probe (diameter: $0.35 \mathrm{~mm}$, uniform geometry) on top of which rests a calibrated cylindrical weight. The probe and weight are mounted inside an aluminum tube. When applied perpendicular to the skin, the probe and weight slide freely inside the tube, thereby maintaining a constant normal force entirely determined by the total mass of the probe and weight. To calibrate the pinprick stimulator, the device was attached to a Scara LS3 4-axis robot (DENSO Products and Services Americas, Inc., CA, USA), and a 6-axis strain-gauge force-torque transducer (Nano 43, ATI Industrial Automation, Inc., Apex, NC, USA) was used to measure the applied normal force with a precision of 1/256 N (Figure 2B). The time course of the normal force generated by applying and maintaining the needle against the force-torque transducer is shown in Figure 2C. Because the elastic properties of the skin and underlying soft tissues may be expected to significantly affect this time course (especially the initial increase in normal force), we also measured the normal force generated by applying the needle against a preparation of skin and muscle tissues taken from the thigh of a chicken (Figure 2C).

In a previous study conducted using a range of pinprick intensities $(16-512 \mathrm{mN}$ ) and a constant $0.25 \mathrm{~mm}$ tip diameter, we found that the brain responses elicited at intermediate pinprick intensities $(64 \mathrm{mN})$ showed the strongest enhancement after the induction of secondary hyperalgesia (van den Broeke et al., 2015). It has been suggested that the strength of activation of mechanical 

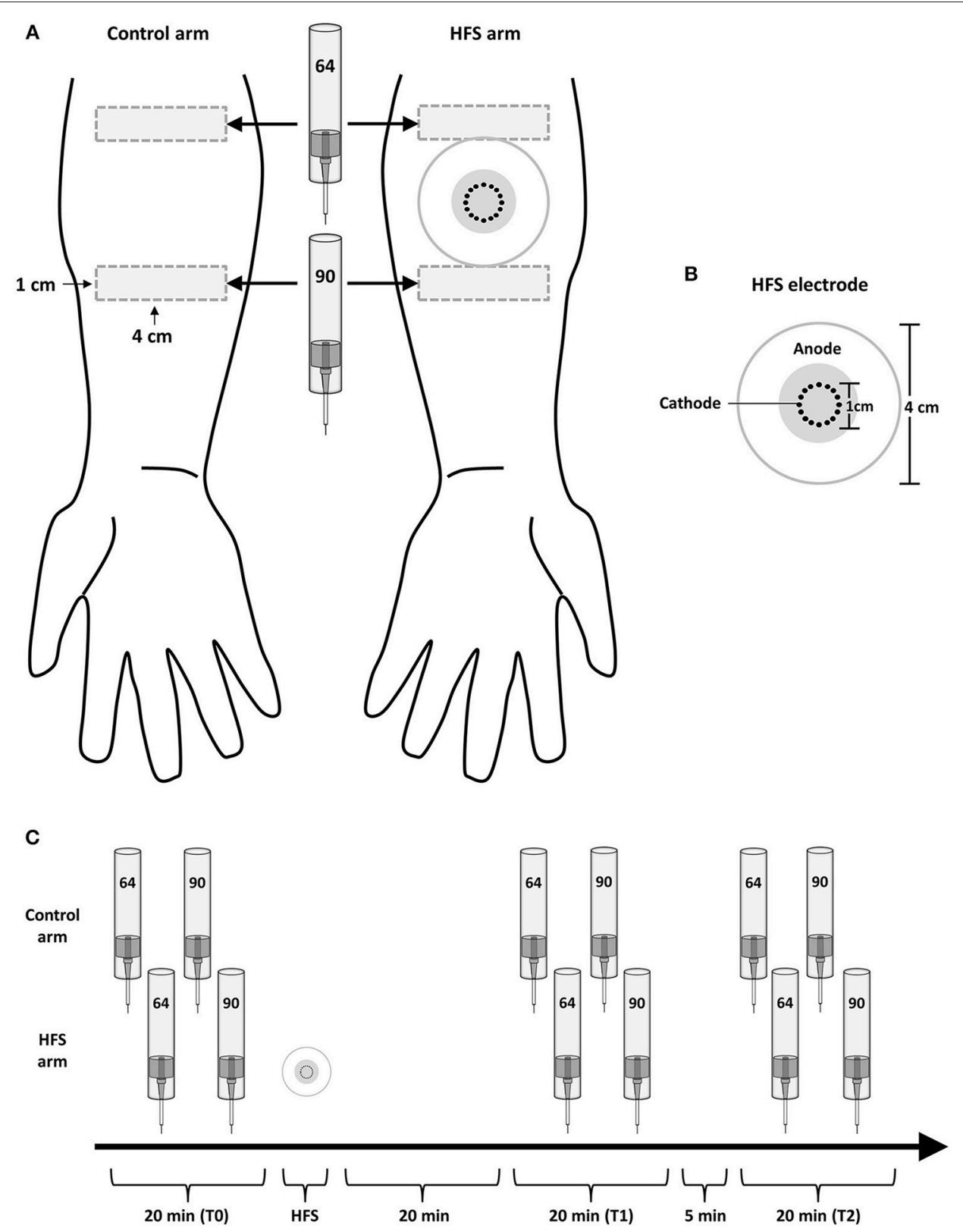

FIGURE 1 | Experimental setup. (A) High frequency electrical stimulation of the skin (HFS) was applied to the left or right volar forearm. Two different intensities of pinprick stimulation (64 and $90 \mathrm{mN}$ ) were applied to the skin surrounding the area onto which HFS was applied as well as to the same skin area on the contralateral arm, which served as control. (B) The electrode used to deliver HFS consisted in 16 blunt stainless steel pins placed in a 10-mm diameter circle (cathode), surrounded by a concentrically-located stainless steel anode. (C) The effect of HFS on the responses elicited by the pinprick stimuli was assessed at three different time points: before HFS (TO), 20 min after HFS (T1) and 45 min after HFS (T2).

nociceptors is not related to the total applied normal force, but to the applied normal force relative to the circumference of the stimulation probe (Garell et al., 1996). In the present study, the tip diameter was $0.35 \mathrm{~mm}$. Therefore, we used two different weights, one matching the total normal force $(64 \mathrm{mN})$, and the other matching the ratio of normal force per circumference ( $90 \mathrm{mN}$, corresponding to $89 \mathrm{mN} / \mathrm{mm}$ ). Each pinprick stimulus was delivered by applying the probe slowly onto the skin and moving the tube downwards and upwards with a total duration of approximately three seconds (see Supplementary Video 1). The reason for doing so is that the time course of the force applied onto the skin at contact onset is not only dependent on the weight of the probe, but also on the speed of the probe and hence its deceleration when contacting the skin. In order to minimize the peak of force at contact onset, the pinprick was applied onto the skin in a slowly fashion. For each intensity of pinprick stimulation (64 and $90 \mathrm{mN}$ ), each arm (HFS and control arm) and each time point (T0, T1, and T2), a total of 


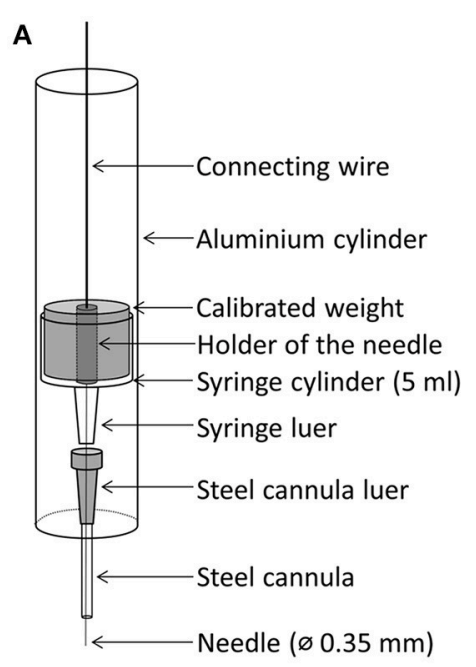

B

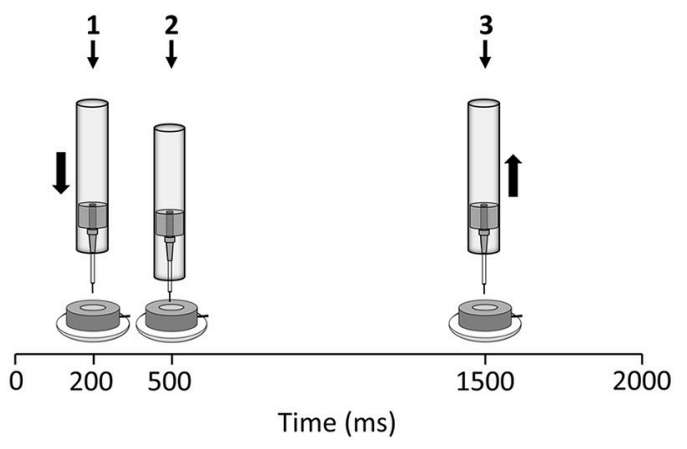

Probe applied against metal surface

C

$64 \mathrm{mN}$

$90 \mathrm{mN}$
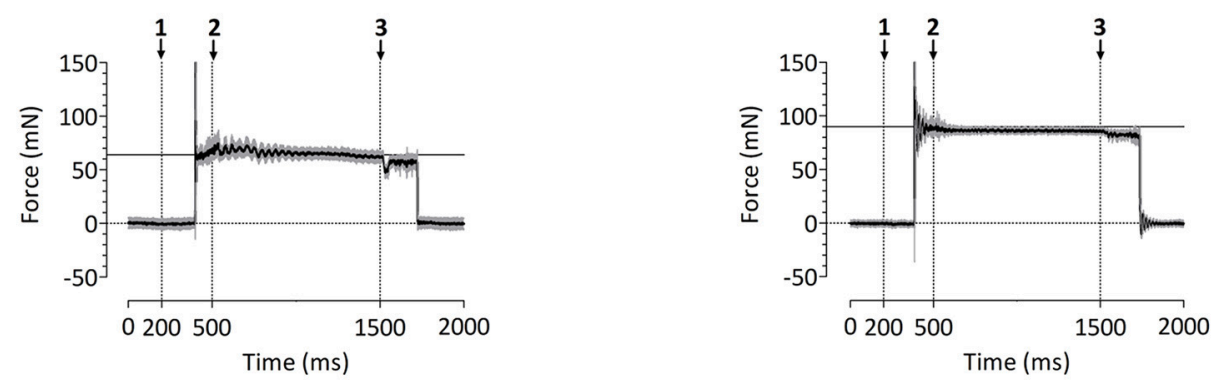

Probe applied against skin overlying soft tissue

$64 \mathrm{mN}$

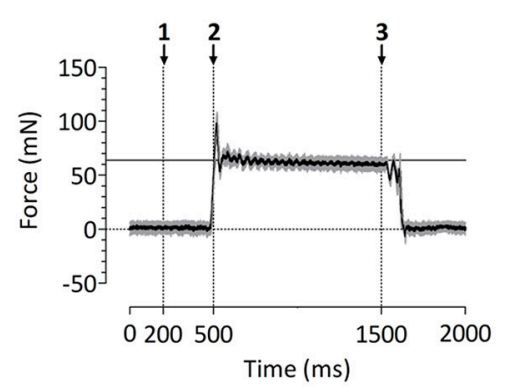

$90 \mathrm{mN}$

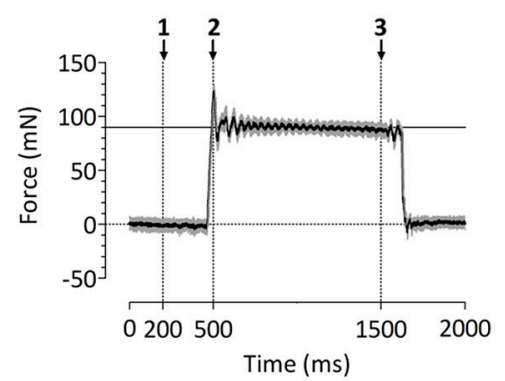

FIGURE 2 | (A) Design of the custom-build mechanical pinprick stimulator used to record pinprick-evoked potentials. (B) Calibration protocol for the mechanical pinprick stimulus. Each trial started with a $200 \mathrm{~ms}$ baseline period. Subsequently, the probe, moved during $300 \mathrm{~ms}$ downwards to the force transducer. Then, the probe was maintained at this position for $1000 \mathrm{~ms}$ and withdrawn back to its initial position during $300 \mathrm{~ms}$. A total of 30 trials were recorded. (C) Time course of the normal force generated against the force transducer with and without overlying soft tissue using the two different pinprick intensities (64 and $90 \mathrm{mN}$ ). The black line shows the average force across the thirty trials. The gray area shows the standard deviation.

20 stimuli were administered. The stimuli were delivered using a random inter-stimulus interval ranging from 3 to $5 \mathrm{~s}$ (self-paced). To avoid sensitization of the stimulated skin, the target of the pinprick stimulus was displaced by the experimenter after each stimulus.

\section{EEG Recording}

The electroencephalogram (EEG) was recorded using 64 actively shielded $\mathrm{Ag}$ - $\mathrm{AgCl}$ electrodes mounted in an elastic electrode-cap and arranged according to the international 1020 system (Waveguard64 cap, Advanced Neuro Technologies, 
The Netherlands). Participants were instructed to sit as still as possible and keep their gaze fixed on a black cross displayed at a distance of approximately $2.0 \mathrm{~m}$. The EEG signals were amplified and digitized using a sampling rate of $1000 \mathrm{~Hz}$ and an average reference (HS64; Advanced Neuro Technologies, The Netherlands). Eye movements were recorded using two surface electrodes placed at the upper-left and lower-right sides of the left eye. Electrode impedances were kept below $20 \mathrm{k} \Omega$. To generate a trigger in the EEG that marked the actual time at which the needle touched the skin we used a high resistance switch triggered by the change in impedance occurring between the probe and a ground electrode placed against the skin at the wrist. A thin layer of conductive gel was used to lower the impedance between the probe and the skin. The delay between the first contact of the skin and trigger generation was almost zero; on average $( \pm S D) 0.046$ $\pm 0.015 \mathrm{~ms}$ as tested in 40 trials using a shortcut circuitry with digitization of the trigger responses.

\section{Intensity of Perception}

The effect of HFS on the intensity of the percept elicited by mechanical pinprick stimulation was assessed by asking participants to rate the intensity of the stimuli on a numerical rating scale (NRS) ranging from 0 (no perception) to 100 (maximal pain), with 50 representing the transition from nonpainful to painful domains of sensation. Within each block of 20 stimuli, the participants were asked to rate pseudo-randomly 10 out of the 20 stimuli directly after receiving the stimulus.

\section{Data Analysis}

\section{Intensity of Perception}

To confirm the successful induction of increased pinprick sensitivity after HFS, we performed a General Linear Model (GLM) repeated measures ANOVA analysis on the intensity of perception ratings using two within-subject factors: time (T0, T1 and T2) and treatment (control vs. HFS arm) for both stimulation intensities (64 and $90 \mathrm{mN}$ ). The assumption of sphericity was tested using Mauchly's test of sphericity. In those cases where the data violated the assumption of sphericity, $F$-values were corrected using the Greenhouse-Geisser procedure. The level of significance was set at $p<0.05$. For post-hoc tests, $p$-values were Bonferroni corrected for the number of tests. The statistical analyses were conducted using SPSS 18 (SPSS Inc., Chicago, IL, USA). The effect of HFS was assessed using the interaction between the factors time and treatment.

\section{EEG Preprocessing}

The EEG signals were analyzed offline using Letswave 6.0 (www.nocions.org/letswave) and MATLAB 2014b (The MathWorks Inc., Natick, MA, USA). After applying a DC correction and a $0.3-30 \mathrm{~Hz}$ band pass zero-phase Butterworth filter to the continuous EEG recordings, the signals were segmented into epochs extending from -500 to $+2000 \mathrm{~ms}$ relative to stimulus onset. Epochs contaminated by eye movements or eye blinks were corrected using an Independent Component Analysis (ICA; Jung et al., 2000). Denoised epochs were then re-referenced to linked mastoids (M1M2). After applying a baseline correction (reference interval: -500 to $0 \mathrm{~ms}$ ), epochs with amplitude values exceeding $\pm 100 \mu \mathrm{V}$ were rejected as these were likely to be contaminated by artifacts. Finally, separate average waveforms were computed for each participant, time point (T0, T1, and T2), stimulation site (HFS and control) and stimulation intensity (64 and $90 \mathrm{mN}$ ). One subject was excluded because the EEG signals contained too many artifacts, which had as consequence that after artifact correction there were no trials left to compute an average waveform.

\section{Pinprick Evoked Potentials (PEPs)}

The effect of HFS on the EEG responses to pinprick stimulation was assessed using a spatio-temporal non-parametric clusterbased permutation approach (Maris and Oostenveld, 2007; Groppe et al., 2011). The advantage of this approach is that it is well suited to analyze long-lasting changes in EEG signals which cannot be summarized as a single peak having a given latency and amplitude, and it provides a simple way to solve the multiple comparison problem related to the point-by-point analysis of EEG signals (Maris and Oostenveld, 2007). First, we computed, for each subject and all electrodes, difference waveforms assessing the change in ERP waveform at T1 vs. T0 and T2 vs. T0 at the control arm (control arm 11 - control $\operatorname{arm}_{\mathrm{T} 0}$; control $\operatorname{arm}_{\mathrm{T} 2}$ - control $\operatorname{arm}_{\mathrm{T} 0}$ ) and at the HFS-treated $\operatorname{arm}\left(\mathrm{HFS} \operatorname{arm}_{\mathrm{T} 1}\right.$ - HFS $\operatorname{arm}_{\mathrm{T} 0}$; HFS $\operatorname{arm}_{\mathrm{T} 2}$ - HFS $\operatorname{arm}_{\mathrm{T} 0}$ ). Then, we performed the cluster-based permutation test on the
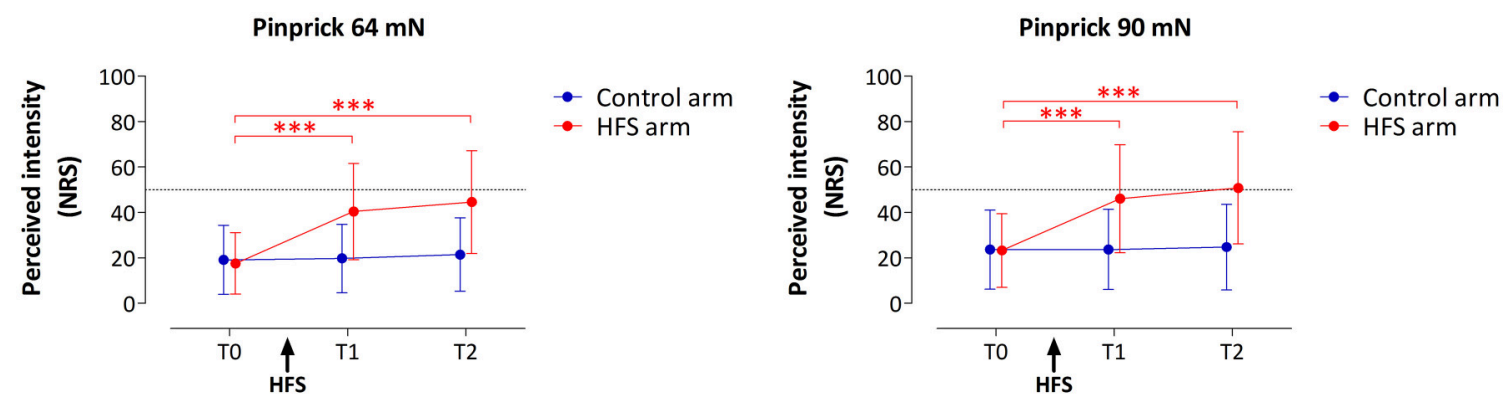

FIGURE 3 | Effect of HFS on intensity of perception elicited by the two pinprick intensities (64 and $90 \mathrm{mN}$ ). Shown are the group-level mean and standard deviation of the numeric rating scale (NRS) scores obtained at the three different time points: before HFS (T0), 20 min after HFS (T1) and 45 min after HFS (T2). Asterisks denote a statistically significant increase of the HFS treated arm compared to T0 ( $p<0.001$, post-hoc paired $t$-test). 
A
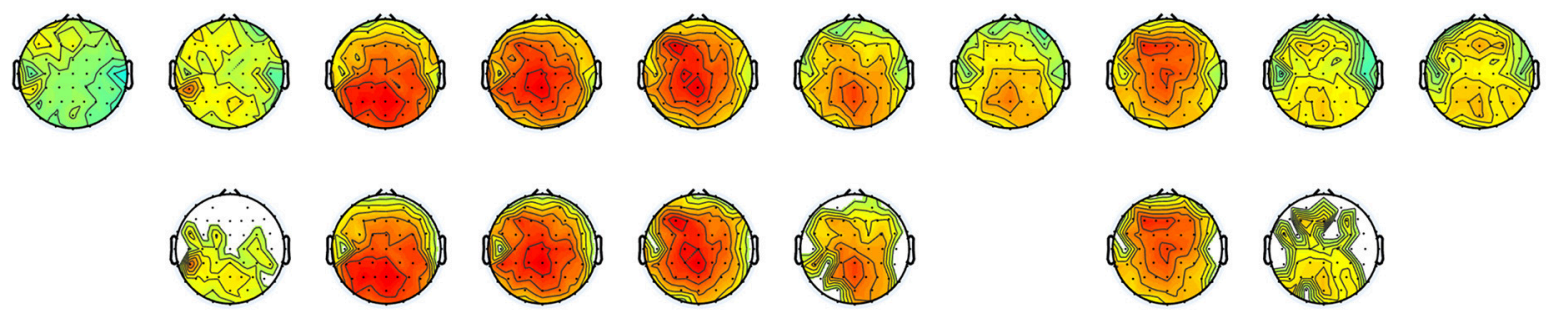

$0.8-1.0 s$

$1.0-1.2$
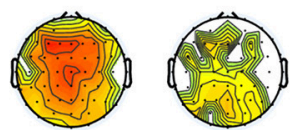

$1.4-1.6 \mathrm{~s}$

$1.6-1.8 \mathrm{~s}$
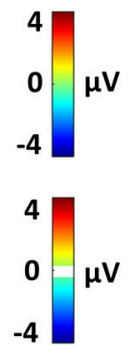

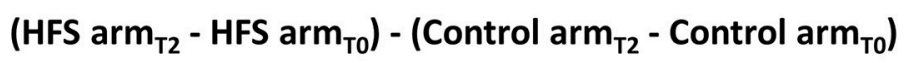
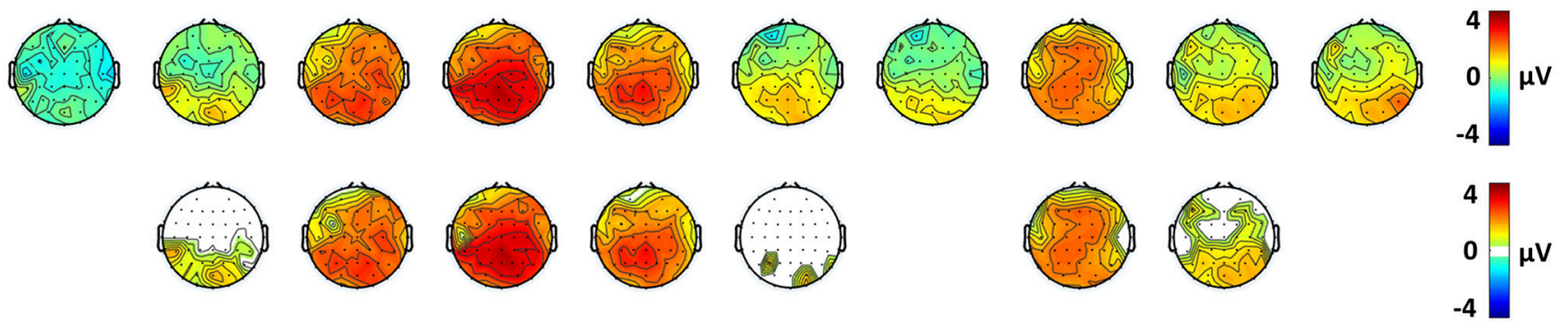

$0-0.2 \mathrm{~s}$

$0.2-0.4 \mathrm{~s}$

$0.4-0.6 s$

$0.6-0.8 s$

$0.8-1.0 \mathrm{~s}$

$1.0-1.2 \mathrm{~s}$

$1.2-1.4 \mathrm{~s}$

$1.4-1.6 \mathrm{~s}$

$1.6-1.8 \mathrm{~s}$

$1.8-2.0 \mathrm{~s}$

Difference waveforms

B<smiles>C1=CCCC=CCCC=CCCC=1</smiles>

Pz

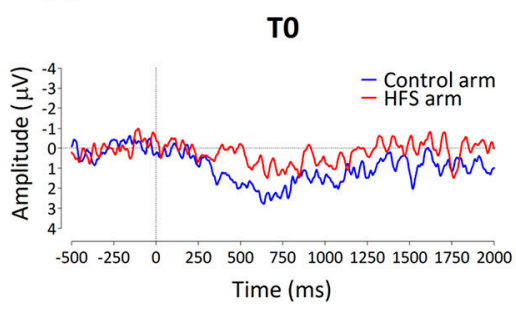

\section{After HFS}

T1 - T0

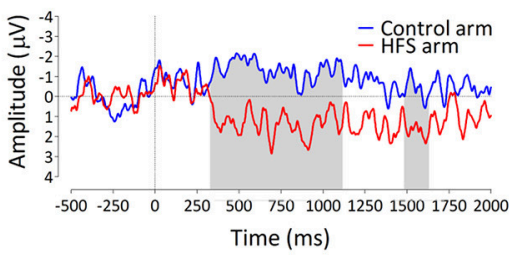

T2 -TO

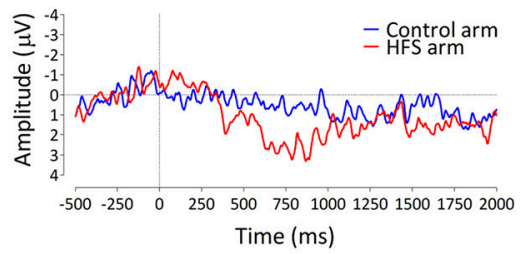

- Control arm
- HFS arm

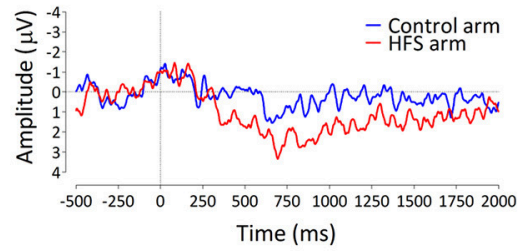

T2

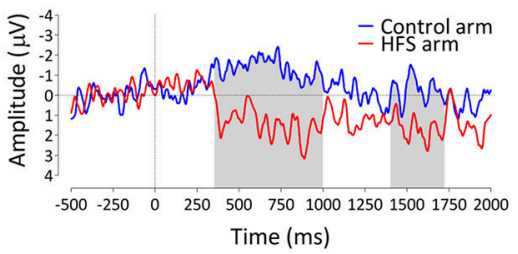

FIGURE 4 | The effect of HFS on PEPs elicited by $64 \mathrm{mN}$ pinprick stimulation. (A) The first row of scalp topographies shows the temporal evolution of the group-level average topography of the difference between the subtracted waves ( $T 1$ minus TO) of both arms for T1. Red denotes an increase of the ERP amplitude at the HFS arm compared to the control arm whereas blue indicates a decrease of ERP amplitude at the HFS arm compared to the control arm. Each topographic plot corresponds to the average amplitude within successive segments of $200 \mathrm{~ms}$. The second row of scalp topographies shows the same topographies, masked by the spatiotemporal pattern of the significant clusters identified using the spatiotemporal cluster-based permutation testing. Masked means that only the time-electrode samples included in the significant clusters are displayed. The third row of scalp topographies shows the temporal evolution of the group- level average topography of the difference between the subtracted waves (T2 minus T0) of both arms for T2. The fourth row of scalp topographies shows those topographies masked using the spatiotemporal pattern of the significant clusters. Scalp topographies were corrected (flipped) according to the side of HFS stimulation. (B) Group-level average ERP waveforms of the signals measured from Pz vs. M1M2, before HFS (TO), 20 min after HFS (T1) and 45 min after HFS treatment (T2) and group-level average difference waveforms (T1-T0 and T2-T0) for the control arm (blue) and the HFS-treated arm (red). Gray shadings indicate the time intervals of the significant clusters shown in (A). 
difference waveforms of both arms, thereby testing the time $x$ treatment interaction. The test included the whole spatial (64 electrodes) and temporal (from 0 to $2000 \mathrm{~ms}$ after stimulus onset) dimensions of the dataset, and consisted of the following steps. First, the difference waveforms obtained at each electrode were compared by means of a point-by-point paired-sample $t$-test. Then, samples above the critical $t$-value for a parametric onesided test that were adjacent in time and space were identified and clustered. Electrodes were considered as adjacent if their distance was $<0.42 \mathrm{~cm}$, based on their standard Cartesian coordinates (Delorme and Makeig, 2004). Considering our 64 channel setup, most electrodes were considered to have four neighbors based on this criterion. An estimate of the magnitude of each cluster was then obtained by computing the sum of the absolute $t$ values constituting each cluster (cluster-level statistic). Random permutation testing (2000 times) of the subject-specific difference waveform of the two arms (performed independently for every subject) was then used to obtain a reference distribution of maximum cluster magnitude. Finally, the proportion of random partitions that resulted in a larger cluster-level statistic than the observed one (i.e., $p$-value) was calculated. Clusters in the observed data were regarded as significant if they had a magnitude exceeding the threshold of the 95th percentile of the permutation distribution (corresponding to a critical alpha-level of 0.05$)$. This analysis was performed independently for both time points ( $\mathrm{T} 1$ and $\mathrm{T} 2$ ) and stimulation intensities (64 and $90 \mathrm{mN})$.

\section{RESULTS}

\section{Intensity of Perception}

HFS induced a clear increase in pinprick sensitivity on the treated arm, as shown by the changes in the intensity of the percept elicited by 64 and $90 \mathrm{mN}$ pinprick stimulation (Figure 3). This was confirmed by repeated-measures ANOVA, which showed a significant time $\times$ treatment interaction for both the $64 \mathrm{mN}$ stimulus $\left[F_{(2,36)}=31.388, p<0.001, \eta^{2}=0.636\right]$ and the $90 \mathrm{mN}$ stimulus $\left[F_{\text {Greenhouse-Geisser }(1.16,20.87)}=24.494, p<0.001, \eta^{2}=\right.$ 0.576].

For both pinprick intensities the univariate within-subject contrasts showed that intensity of perception was significantly enhanced at the HFS-treated arm, both at T1 [64 $m N: F_{(1,18)}$ $=37.962, p<0.001, \eta^{2}=0.678 ; 90 m N: F_{(1,18)}=25.421, p$ $\left.<0.001, \eta^{2}=0.585\right]$ and at T2 $\left[64 m N: F_{(1,18)}=38.131, p<\right.$

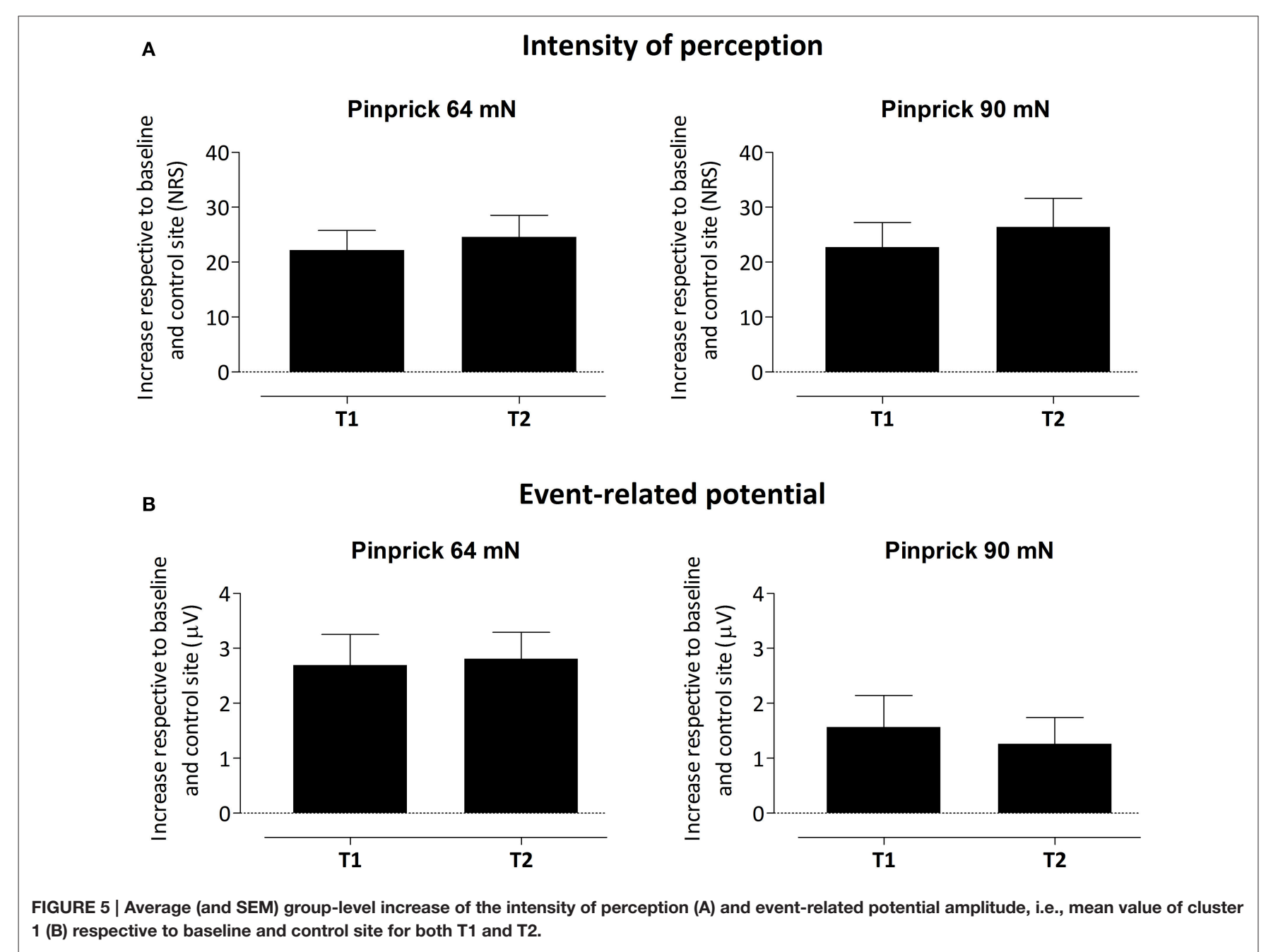


$0.001, \eta^{2}=0.679 ; 90 \mathrm{mN}: F_{(1,18)}=26.012, p<0.001, \eta^{2}=$ 0.591]. Post-hoc tests showed, for both intensities, a statistically significant increase of perceived intensity at the treated arm, both at T1 [64 $\mathrm{mN}$ : paired $t$-test; $t_{(18)}=-6.436, p<0.001 ; 90 \mathrm{mN}$ : $\left.t_{(18)}=-5.060, p<0.001\right]$ and at T2 [64 $m N: t_{(18)}=-6.402, p<$ $\left.0.001 ; 90 m N: t_{(18)}=-5.245, p<0.001\right]$.

\section{Pinprick Evoked Brain Potentials (PEPS) PEPs Elicited by $64 \mathrm{mN}$ Pinprick Stimulation}

The group-level average waveforms of the PEPs elicited by the $64 \mathrm{mN}$ stimulation are shown in Figure 4. The elicited response consisted mainly of a long-lasting positive wave, maximal at midline central-posterior electrodes. The results of the cluster-based permutation test performed on the ERP difference waveforms (after vs. before HFS) of both arms (control vs. HFS) for both time points (T1 and T2) revealed two positive clusters having a $p$-value smaller than the critical alpha level of 0.05 , both at T1 and at T2 (Figure 4). At T1, the first cluster extended between 326 and $1122 \mathrm{~ms}$ after stimulus onset $(p<$ 0.001 ), and the second cluster extended between 1481 and 1638 $\mathrm{ms}(p<0.05)$. At T2, the first cluster extended between 350 and $1005 \mathrm{~ms}(p<0.001)$, and the second cluster extended between 1398 and $1728 \mathrm{~ms}(p<0.05)$. Both at T1 and at T2, the first cluster was widely distributed but maximal at central-posterior scalp electrodes, whereas the second cluster displayed a more centralfrontal distribution (Figure 4A). These two clusters demonstrate a statistically significant difference in PEP amplitude between the two arms, which was present both $20 \mathrm{~min}$ (T1) and $45 \mathrm{~min}$ (T2) after applying HFS.

To assess whether the difference in PEP amplitude between the two arms after HFS was due to an increase of PEP amplitude at the HFS-treated arm, a decrease of PEP amplitude at the control arm, or both, we performed post-hoc tests (paired $t$-tests, twosided, Bonferroni corrected) on the individual mean amplitude values calculated within cluster 1 at electrode Pz. The paired $t$-tests revealed both (1) at the HFS-treated arm, a significant increase in mean PEP amplitude at T1 compared to T0 $\left[t_{(18)}=\right.$ $-3.088, p=0.006]$ and T2 compared to T0 $\left[t_{(18)}=-3.024, p=\right.$ 0.007 ] and (2) at the control arm, a significant decrease in mean PEP amplitude at T1 compared to T0 $\left[t_{(18)}=3.432, p=0.003\right]$ and at T2 compared to T0 $\left[t_{(18)}=3.133, p=0.006\right]$. Figure 5 shows the average (and SEM) group-level increase in perceived intensity and ERP amplitude (mean value cluster 1) respective to baseline and control site for T1 and T2.

\section{PEPs Elicited by 90 mN Pinprick Stimulation}

The group-level average waveforms of the PEPs elicited by 90 $\mathrm{mN}$ stimulation are shown in Figure 6. Such as for the 64 $\mathrm{mN}$ stimulus, the elicited response consisted mainly of a longlasting positive wave, maximal at central-posterior electrodes. The permutation testing did not identify any cluster having a $p$-value smaller than the critical alpha level of 0.05 .

\section{DISCUSSION}

The present study shows, for the first time, that the increase in brain response elicited by the $64 \mathrm{mN}$ mechanical pinprick stimulation in the area of secondary hyperalgesia follows the same time course as the increase in pinprick sensitivity, both being present 20 and 45 min after applying HFS.

\section{T2: A Crucial Time Point}

Performing a second post measurement 45 min after applying HFS is crucial as it allows distinguishing unspecific shortlasting from specific long-lasting effects of HFS. The increase in PEP positivity followed the long-lasting time course of the increase in pinprick sensitivity. Indeed, both were present 20 and $45 \mathrm{~min}$ after HFS. Importantly, this long-lasting increase

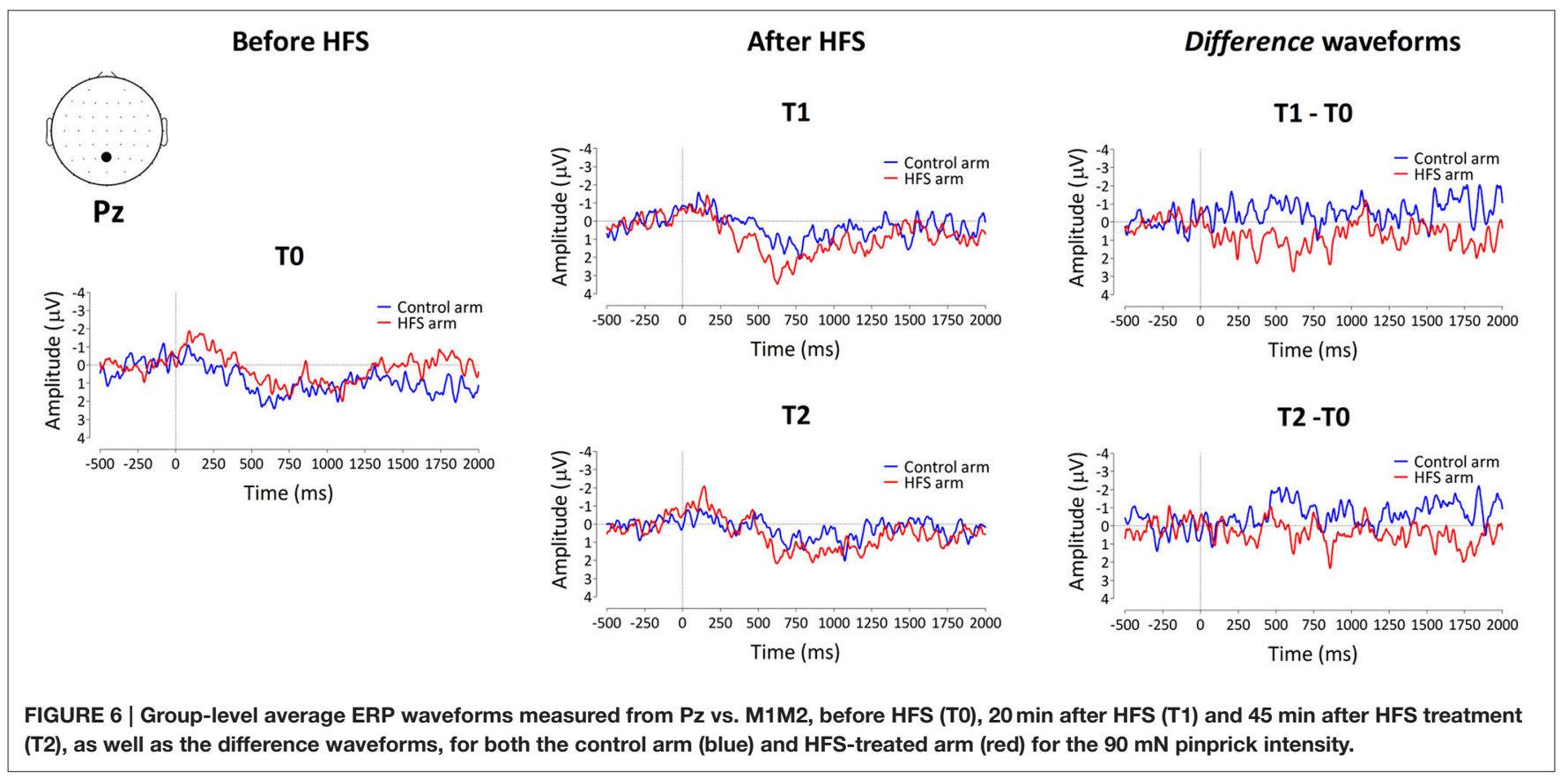


in PEP positivity is in striking contrast with the shortlasting enhancement of EEG responses to brief non-nociceptive vibrotactile and thermal stimulation that have been reported in previous studies (van den Broeke and Mouraux, 2014a,b). Indeed, in these studies, the EEG enhancements were observed 20 min after HFS, but disappeared completely 45 min after HFS. The fact that HFS appears to produce various effects with distinct time courses suggests the existence of multiple mechanisms. In addition to a long-lasting central sensitization of mechanical nociceptive pathways, leading to a selective increase in pinprick sensitivity, HFS appears to also produce short-lasting and modality unspecific changes, which could be the consequence of higher-order cognitive mechanisms. For example, delivering HFS on one arm could lead to a sustained orientation of spatial attention toward the treated arm.

\section{Increased Pinprick Sensitivity}

Such as in our previous studies (van den Broeke and Mouraux, 2014a,b), the perception elicited by the pinprick stimuli at baseline were not qualified as painful. Therefore, the increased pinprick sensation observed after applying HFS does not satisfy the current definition of "secondary hyperalgesia" proposed by the International Association for the Study of Pain (IASP), which restricts the term hyperalgesia to "increased pain from a stimulus that normally provokes pain" (Loeser and Treede, 2008). However, studies using microneurography have clearly demonstrated that punctate probes like the ones used in the present study preferentially activate mechanosensitive nociceptors when applied against the skin (Garell et al., 1996; Slugg et al., 2000, 2004). Moreover, a study comparing the perceptual pain thresholds in human volunteers with the thresholds for nociceptors in animals using the same mechanical punctate probes, suggests that the non-painful sharp pricking sensation is mediated by mechanosensitive nociceptors (Greenspan and McGillis, 1991). Therefore, even though the mechanical stimuli elicited, at baseline, a pinprick percept that was not qualified as painful, the increased perception to these stimuli when delivered in the area of increased pinprick sensitivity is most probably related to a central sensitization of mechanical nociceptive pathways.

\section{PEPs Elicited from the Area of Increased Pinprick Sensitivity}

In our previous study, a range of pinprick intensities (16$512 \mathrm{mN}$ ) was used to characterize the effect of stimulation intensity on the PEPs elicited by stimulation before vs. after and within vs. outside the area of secondary hyperalgesia (van den Broeke et al., 2015). We observed a nonlinear (inverted

\section{REFERENCES}

Ali, Z., Meyer, R. A., and Campbell, J. N. (1996). Secondary hyperalgesia to mechanical but not heat stimuli following capsaicin injection in hairy skin. Pain 68, 401-411. doi: 10.1016/S0304-3959(96)03199-5

Baumann, T. K., Simone, D. A., Shain, C. N., and LaMotte, R. H. (1991). Neurogenic hyperalgesia: the search for the primary cutaneous afferent fibers that contribute to capsaicin-induced pain and hyperalgesia. J. Neurophysiol. 66, $212-227$.
U-shape) relationship between stimulation intensity and the increase in PEPs elicited from the area of secondary hyperalgesia. The strongest (and only significant) increase in PEP was elicited by the intermediate $64 \mathrm{mN}$ stimulation intensity. The current results are in agreement with that study. Indeed, we observed a significant increase in PEP for the $64 \mathrm{mN}$ stimulation, but not for the $90 \mathrm{mN}$ stimulation. At present, we can only speculate about why there is no significant increase in PEP amplitude for the $90 \mathrm{mN}$ and higher stimulation intensities. One possibility could be a ceiling effect: at $90 \mathrm{mN}$, the PEPs recorded in the absence of sensitization could reach an upper limit above which no further increase in PEP magnitude can be observed. Another possibility could be that the higher levels of arousal elicited by higher stimulation intensities could, in some way, inhibit the cortical responses underlying the increase in PEP magnitude. Supporting this hypothesis, a previous study observed a similar non-linear relationship between the enhancement of a positive wave elicited by known vs. novel images and different levels of arousal in an emotional recognition task. The largest difference in amplitude, peaking around $300 \mathrm{~ms}$ after stimulation onset, was observed for intermediate levels of arousal (Schaefer et al., 2009).

\section{AUTHOR CONTRIBUTIONS}

$\mathrm{EV}, \mathrm{JL}$, and $\mathrm{AM}$ were involved in the conception and design of the study. EV, JL, and GH collected and analyzed the data. EV, JL, $\mathrm{GH}$, and AM interpreted the data and drafted the manuscript. All authors have approved the final version of the manuscript.

\section{FUNDING}

EV is supported by the Belgian National Foundation for Scientific Research (Mandat d'impulsion scientifique FNRS 14613969) and the ERC "Starting Grant" (PROBING PAIN 336130). AM is supported by the ERC "Starting Grant" (PROBING PAIN 336130). GH is supported by the Belgian National Foundation for Scientific Research (Mandat d'impulsion scientifique FNRS 14613969), and JL is supported by the ERC "Starting Grant" (PROBING PAIN 336130).

\section{SUPPLEMENTARY MATERIAL}

The Supplementary Material for this article can be found online at: http://journal.frontiersin.org/article/10.3389/fnhum. 2016.00531

Supplementary Video 1 | Demonstration pinprick stimulation.

Delorme, A., and Makeig, S. (2004). EEGLAB: an open source toolbox for analysis of single-trial EEG dynamics including independent component analysis. J. Neurosci. 134, 9-21. doi: 10.1016/j.jneumeth.2003.10.009

Garell, P. C., McGillis, S. L. B., and Greenspan, J. D. (1996). Mechanical response properties of nociceptors innervating feline hairy skin. J. Neurophysiol. 75, 1177-1189.

Greenspan, J. D., and McGillis, S. L. B. (1991). Stimulus features relevant to the perception of sharpness and mechanically evoked cutaneous pain. Somatosens. Mot. Res. 8, 137-147. doi: 10.3109/08990229109144738 
Groppe, D. M., Urbach, T. P., and Kutas, M. (2011). Mass univariate analysis of event-related brain potentials/fields I: a critical tutorial review. Psychophysiology 48, 1711-1725. doi: 10.1111/j.1469-8986.2011. 01273.x

Henrich, F., Magerl, W., Klein, T., Greffrath, W., and Treede, R. D. (2015). Capsaicin-sensitive C-and A-fibre nociceptors control long-term potentiation-like pain amplification in humans. Brain 138, 2505-2520. doi: 10.1093/brain/awv108

Jung, T. P., Makeig, S., Humphries, C., Lee, T. W., McKeown, M. J., Iragui, V., et al. (2000). Removing electroencephalographic artifacts by blind source separation. Psychophysiology 37, 163-178. doi: 10.1111/1469-8986.3720163

LaMotte, R. H., Shain, C. N., Simone, D. A., and Tsai, E.-F. (1991). Neurogenic hyperalgesia: psychophysical studies of underlying mechanisms. J. Neurophysiol. 66, 190-211.

Loeser, J. D., and Treede, R. D. (2008). The Kyoto protocol of IASP basic pain terminology. Pain 137, 473-477. doi: 10.1016/j.pain.2008.04.025

Magerl, W., Fuchs, P. N., Meyer, R. A., and Treede, R. D. (2001). Roles of capsaicininsensitive nociceptors in cutaneous pain and secondary hyperalgesia. Brain 124, 1754-1764. doi: 10.1093/brain/124.9.1754

Magerl, W., Wilk, S. H., and Treede, R. D. (1998). Secondary hyperalgesia and perceptual wind-up following intradermal injection of capsaicin in humans. Pain 74, 257-268. doi: 10.1016/S0304-3959(97)00177-2

Maris, E., and Oostenveld, R. (2007). Nonparametric statistical testing of EEG- and MEG-data. J. Neurosci. Meth. 164, 177-190. doi: 10.1016/j.jneumeth.2007.03.024

Nicholls, M. E., Thomas, N. A., Loetscher, T., and Grimshaw, G. M. (2013). The Flinders Handedness survey (FLANDERS): a brief measure of skilled hand preference. Cortex 49, 2914-2926. doi: 10.1016/j.cortex.2013.02.002

Pfau, D. B., Klein, T., Putzer, D., Pogatzki-Zahn, E. M., Treede, R. D., and Magerl, W. (2011). Analysis of hyperalgesia time courses in humans after painful electrical high-frequency stimulation identifies a possible transition from early to late LTP-like pain plasticity. Pain 152, 1532-1539. doi: 10.1016/j.pain.2011.02.037

Raja, S. N., Campbell, J. N., and Meyer, R. A. (1984). Evidence for different mechanisms of primary and secondary hyperalgesia following heat injury to the glabrous skin. Brain 107, 1179-1188. doi: 10.1093/brain/107.4.1179

Schaefer, A., Fletcher, K., Pottage, C. L., Alexander, K., and Brown, C. (2009). The effects of emotional intensity on ERP correlates of recognition memory. Neuroreport 20, 319-324. doi: 10.1097/WNR.0b013e3283 $229 b 52$

Simone, D. A., Sorkin, L. S., Oh, U., Chung, J. M., Owens, C., LaMotte, R. H., et al. (1991). Neurogenic hyperalgesia: central neural correlates in responses to spinothalamic tract neurons. J. Neurophysiol. 66, 228-246.

Slugg, R. M., Campbell, J. N., and Meyer, R. A. (2004). The population response of A- and C-fiber nociceptors in monkey encodes high-intensity mechanical stimuli. J. Neurosci. 24, 4649-4656. doi: 10.1523/JNEUROSCI.0701-04.2004

Slugg, R. M., Meyer, R. A., and Campbell, J. N. (2000). Response of cutaneous A- and C-fiber nociceptors in the monkey to controlled-force stimuli. $J$. Neurophysiol. 83, 2179-2191.

van den Broeke, E. N., and Mouraux, A. (2014a). High frequency electrical stimulation of the human skin induces heterotopical mechanical hyperalgesia, heat hyperalgesia and enhanced responses to non-nociceptive vibrotactile input. J. Neurophysiol. 111, 1564-1573. doi: 10.1152/jn.00651.2013

van den Broeke, E. N., and Mouraux, A. (2014b). Enhanced brain responses to C-fiber input in the area of secondary hyperalgesia induced by high frequency electrical stimulation of the skin. J. Neurophysiol. 112, 2059-2066. doi: 10.1152/jn.00342.2014

van den Broeke, E. N., Mouraux, A., Groneberg, A. H., Pfau, D. B., Treede, R. D., and Klein, T. (2015). Characterizing pinprick evoked brain potentials before and after experimentally induced secondary hyperalgesia. J. Neurophysiol. 114 2672-2681. doi: 10.1152/jn.00444.2015

Ziegler, E. A., Magerl, W., Meyer, R. A., and Treede, R. D. (1999). Secondary hyperalgesia to punctate mechanical stimuli: central sensitization to A-fibre nociceptor input. Brain 122, 2245-2257. doi: 10.1093/brain/122.12.2245

Conflict of Interest Statement: The authors declare that the research was conducted in the absence of any commercial or financial relationships that could be construed as a potential conflict of interest.

Copyright (C) 2016 van den Broeke, Lambert, Huang and Mouraux. This is an openaccess article distributed under the terms of the Creative Commons Attribution License (CC BY). The use, distribution or reproduction in other forums is permitted, provided the original author(s) or licensor are credited and that the original publication in this journal is cited, in accordance with accepted academic practice. No use, distribution or reproduction is permitted which does not comply with these terms. 